\title{
Adaptive asynchronous algorithm for fringe pattern demodulation
}

\author{
J. A. Gómez-Pedrero, ${ }^{1, \star}$ J. A. Quiroga, ${ }^{1}$ and M. Servín ${ }^{2}$ \\ 'Optics Department, Universidad Complutense de Madrid, Facultad de Ciencias Físicas, \\ Ciudad Universitaria, s/n 28040 Madrid, Spain \\ ${ }^{2}$ Centro de Investigaciones en Óptica, Loma del Bosque No. 115 Col. Lomas del Campestre, \\ C.P. 37150 A.P. 1-948 León, Guanajuato, México \\ ${ }^{*}$ Corresponding author: jagomezp@ fis.ucm.es
}

Received 27 March 2008; revised 23 May 2008; accepted 22 June 2008; posted 23 June 2008 (Doc. ID 94317); published 17 July 2008

\begin{abstract}
We present a spatial adaptive asynchronous algorithm for fringe pattern demodulation. The proposed algorithm is based on the standard five-step asynchronous method with the one modification that we select the best sample spacing for each point of the fringe pattern. As we show, the frequency response of any asynchronous method depends on the sample spacing. This interesting behavior is used to select the best sample spacing as the one that gives the biggest response for each location. The overall result is a spatial demodulation algorithm with an improved frequency response compared to the existing ones. We show the feasibility of the proposed method with theoretical analysis as well as experimental results. (c) 2008 Optical Society of America
\end{abstract}

OCIS codes: $\quad 100.2650,120.5050,120.4120,120.5410$.

\section{Introduction}

Many optical measurement techniques rely on the extraction of the phase of a fringe pattern in order to determine the quantity to be measured [1]. Examples of these techniques are found in holography, interferometry, moiré methods, and photoelasticity [1-3]. To extract the information codified in a fringe pattern, researchers have developed many procedures that are known in the literature as phase evaluation methods (PEMs) [1]. In recent years, considerable efforts have been devoted to the search for fully automatic PEMs that would be faster, more reliable, and more robust than the existing ones.

Among the existing PEMs there are a large number of techniques that can be classified as phase-sampling algorithms (PSAs) [1-3]. The goal of these PSAs is to extract the phase of a fringe pattern from a set of irradiance samples $\left\{I_{j}\right\}_{j=1,2, \ldots N}$ that differ only on a phase shift between consecutive samples. If we as-

0003-6935/08/213954-08\$15.00/0

(C) 2008 Optical Society of America sume a sinusoidal model for the fringe pattern [1], the irradiance of a sample is given by the following equation:

$$
I_{j}=a+b \cos \left(\varphi+\alpha_{j}\right), \quad j=1,2, \ldots, N,
$$

where $a$ is the background, $b$ is the fringe modulation, $\varphi$ is the fringe pattern phase, and $a_{j}$ is the phase jumps. PSA techniques can be classified according to the nature of the phase jumps. We will talk of synchronous PSAs if all the phase jumps $\left\{\alpha_{j}\right\}_{j=1,2, \ldots N}$ are known [2,3]. Otherwise, if the phase jumps are unknown, then an asynchronous PSA must be employed to demodulate the phase [2]. A wide variety of asynchronous phase sampling algorithms (also named self-calibrating PSAs [4] or compensated relative phase shift PSAs [1]) can be found in the literature, such as the five-step algorithm $[4,5]$ or the four-step algorithm attributed to Carré [6]. These algorithms usually assume that locally the phase jump between samples is constant with a spatial frequency $\omega$, that is, $\alpha_{j}=\omega j$. Therefore, introducing $\omega$ as an additional unknown, the phase can be demodulated through 
algebraically exact expressions. These asynchronous (or self-calibrating) PSAs return the wrapped phase $W[\varphi]$ and an estimation of the phase sampling frequency $\omega$ as algebraic functions of the set of irradiance samples. For example, the wrapped phase and frequency $\omega$ of Carré's algorithm are given by

$$
\begin{array}{r}
W[\varphi]= \\
\arctan \frac{\sqrt{\left[3\left(I_{2}-I_{3}\right)-\left(I_{1}-I_{4}\right)\right]\left[\left(I_{1}-I_{4}\right)+\left(I_{2}-I_{3}\right)\right]}}{\left(I_{2}+I_{3}\right)-\left(I_{1}+I_{4}\right)}, \\
\tan ^{2} \omega=\frac{3\left(I_{2}-I_{3}\right)-\left(I_{1}-I_{4}\right)}{\left(I_{1}-I_{4}\right)+\left(I_{2}-I_{3}\right)} . \quad(2 \mathrm{a})
\end{array}
$$

This scheme is valid for all the asynchronous PSAs, so for any of these algorithms, $\tan \varphi$ is calculated as the quotient of two functions, $u\left(I_{j}\right)$ and $v\left(I_{j}\right)$. It is well known that in the presence of noise the phase extraction reliability depends on the frequency response $m(\omega)[\underline{3}, 7]$ defined as

$$
m(\omega)=\sqrt{u^{2}+v^{2}} .
$$

According to [3] , Eq. (2a) renders reliable results when $m(\omega)$ is maximum. For example, in the case of Carré's algorithm, frequency response is given by

$$
m(\omega)=|2 \cos 3 \omega-\cos \omega| .
$$

So, the optimum working point is a $\omega=55 \%$ sample, which is the maximum of Eq. (4). This coincides with the well-known result that the samples in the Carré method should be separated by $110^{\circ}$ to obtain optimum performance in front of additive noise.

In general, for any asynchronous method, we will always find an optimum frequency for which the response is maximum. Therefore, a desirable goal in the design of asynchronous PSA is to find algorithms with the greatest frequency bandwidth.

In a previous paper, Crespo et al. [7] have developed a method to design an asynchronous algorithm optimized for wide frequency response using a technique based on Fourier analysis. Although this technique allows the designing of asynchronous PSAs with arbitrary frequency response, the resulting algorithms are not algebraically exact. This means that, although the algorithms present optimum noise rejection properties, the phase is affected by algebraic errors.

To overcome this problem, we propose in this paper a new adaptive asynchronous PSA based on the standard five-step asynchronous algorithm [4,5]. With the proposed adaptive algorithm, we get an improved frequency response while retaining the exact algebraic nature of the algorithm. Therefore, in the spatial domain, we can demodulate fringe patterns with high frequency dynamic range. We must point out that the idea presented can be easily adapted to similar asynchronous algorithms, such as Carré's. Alternative techniques found in the literature are the windowed Fourier transform [8] and wavelet transform analysis $[9,10]$. In these techniques, a space frequency analysis is performed locally that increments the dimensions of the problem to be solved. The proposed method, although adaptive in nature, retains the simplicity of the classical phase-sampling techniques.

In Section 2 we present the theoretical foundations of the method. Experimental results are shown in Section 3 and, finally, conclusions are given at the end of the paper.

\section{Adaptive Asynchronous Algorithm for Fringe Pattern Demodulation}

For the sake of clarity, we will make a one-dimensional (1D) analysis, but our results can be extended straightforwardly to the two-dimensional (2D) case. In the case of the five-step asynchronous method, the irradiance samples around the point of interest $(j=3)$ are given by

$$
I_{j}=a+b \cos [\varphi+\omega(j-3)], \quad j=1 \ldots 5,
$$

so the phase can be demodulated by $[\underline{5}, \underline{3}]$

$$
\tan \varphi=\frac{u}{v}=\frac{\operatorname{sign}\left(I_{2}-I_{4}\right) \sqrt{4\left(I_{2}-I_{4}\right)^{2}-\left(I_{1}-I_{5}\right)^{2}}}{2 I_{3}-I_{1}-I_{5}},
$$

where sign() stands for the sign function. From Eqs. ( and (ㅁ), $u$ and $v$ are given as []

$$
\begin{aligned}
& u=4 b \sin \varphi \sin ^{2} \omega, \\
& v=4 b \cos \varphi \sin ^{2} \omega .
\end{aligned}
$$

Therefore, by Eq. (ㄴ) $)$ the frequency response is given by

$$
m(\omega)=4 b \sin ^{2}(\omega) .
$$

According to Eq. (8), the frequency response depends on the spatial frequency $\omega$. It can be demonstrated that the response reaches its maximum when $\omega=\pi / 2 \mathrm{rad} /$ sample, which is the well-known [5,7] optimum working point for the five-step asynchronous algorithm. Conversely, frequency response is very low for the extreme frequencies, when $\omega$ is close to 0 and $\pi$ $\mathrm{rad} /$ sample, respectively. For a real fringe pattern, this means that unless the spatial frequency is close to $\omega=\pi / 2 \mathrm{rad} / \mathrm{sample}$, the reliability of the recovered phase will be low.

In the case of a phase pattern with a dominant spatial frequency of $\pi / 4 \mathrm{rad} /$ sample, the straightforward solution will be the spatial decimation by 1 pixel of the irradiance signal, so the spatial frequency becomes $\pi / 2 \mathrm{rad} / \mathrm{sample}$. After this, we would apply the fivestep algorithm. Decimation by 1 pixel consists of throwing away one of every two samples. However, in real cases, the dominant spatial frequency is not 
known or, simply, the fringe pattern is not monochromatic. Therefore, this simple decimation strategy must be changed to make it local and adaptive.

The irradiance samples obtained with a decimation of $\Delta-1$ pixel will be

$$
\begin{aligned}
& I_{1}=a+b \cos (\varphi-2 \omega \Delta), \\
& I_{2}=a+b \cos (\varphi-\omega \Delta), \\
& I_{3}=a+b \cos (\varphi), \\
& I_{4}=a+b \cos (\varphi+\omega \Delta), \\
& I_{5}=a+b \cos (\varphi+2 \omega \Delta) .
\end{aligned}
$$

If we set $\Delta=1$, we have the standard five-step algorithm; setting $\Delta=2$ is equivalent to decimation by one pixel, setting $\Delta=3$ decimates by two pixels, and so on. As can be seen in Eq. (9), the decimation is equivalent to change $\omega$ by $\omega \Delta$. Therefore, the frequency response for this signal would be

$$
m(\Delta, \omega)=4 b \sin ^{2}(\omega \Delta) .
$$

In Fig. 1, we have represented the response $m(\Delta, \omega)$ as a function of the spatial frequency $\omega$ for different values of $\Delta$. These plots show explicitly the correctness of our previously intuitive discussion about decimation. In effect, for $\Delta=2$, the maximum response is obtained at $\pi / 4 \mathrm{rad} / \mathrm{sample}$ instead of $\pi / 2 \mathrm{rad} /$ sample. Moreover, another maximum response is obtained at $3 \pi / 4 \mathrm{rad} / \mathrm{sample}$, which is a nonintuitive and very interesting result. Therefore, as it is show in Fig. 1, changing the decimation factor $\Delta$ allows the asynchronous method tuning for different spatial frequencies in the range $[0, \pi]$.

The adaptive demodulation method proposed consists of selecting the best decimation factor $\Delta$ for each location. The best decimation factor would be

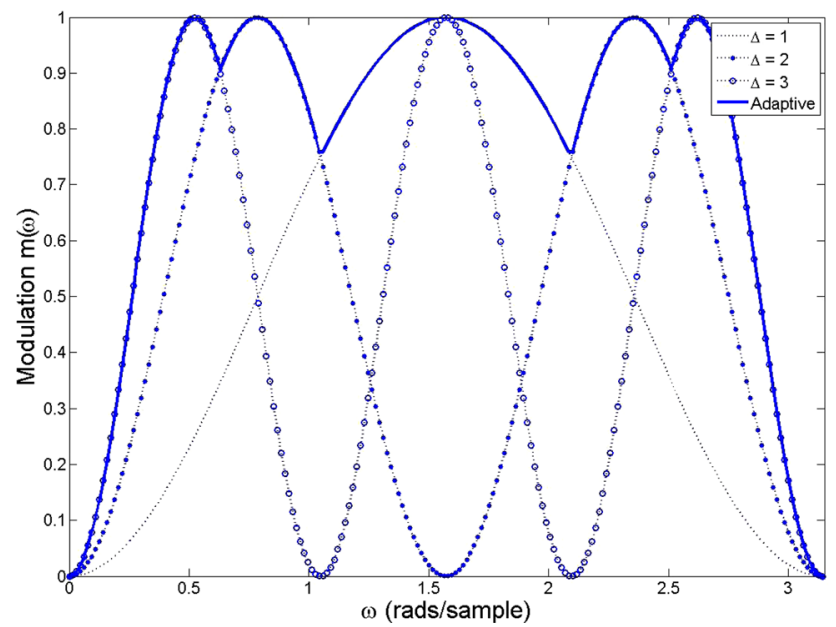

Fig. 1. (Color online) Theoretical response function for the adaptive five-step asynchronous algorithm as function of the local frequency (continuous line) compared with the response of the standard five-step asynchronous algorithm decimated by 0 $(\Delta=1), 1(\Delta=2)$, and $2(\Delta=3)$ pixels. the one that maximizes the frequency response $m(\Delta, \omega)$ for each point. In this way, for the three decimation factors in Fig. 1, the response of the adaptive method will be the thick line plot. Mathematically, as we vary $\Delta$, we get a set of wrapped phase values $W[\varphi(\Delta)], \Delta=1 \ldots N$, each one with a corresponding frequency response $m(\Delta)$. Then, the optimum decimation factor $\delta$ will be one that leads to a maximum frequency response, that is,

$$
\delta=\{\Delta \mid m(\Delta)=\max [m(1), m(2), \ldots m(N)]\} .
$$

And the phase for this point will be $W[\varphi(\delta)]$. The procedure is repeated for every point of the fringe pattern.

To summarize, the proposed algorithm can be specified in the following steps (see flow chart depicted in Fig. 2):

1. For a given point and for each value of the decimation factor $\Delta$ in a given range $[0, N]$, we calculate the wrapped phase by Eq. ( $\underline{6})$ and the frequency response by Eq. (3).

2. Determine the optimum decimation factor $\delta$ using the condition stated in Eq. (11).

3 . The wrapped phase $W[\varphi]$ and modulation $m$ are the ones corresponding to $\delta$.

4. Repeat steps 1 to 3 for every point of the fringe pattern.

\section{Results}

To illustrate the performance of the proposed method, we have first made a numerical experiment. We have generated a horizontal chirp fringe pattern given by

$$
I(x, y)=100+60 \cos [\varphi(x)+r(x, y)],
$$

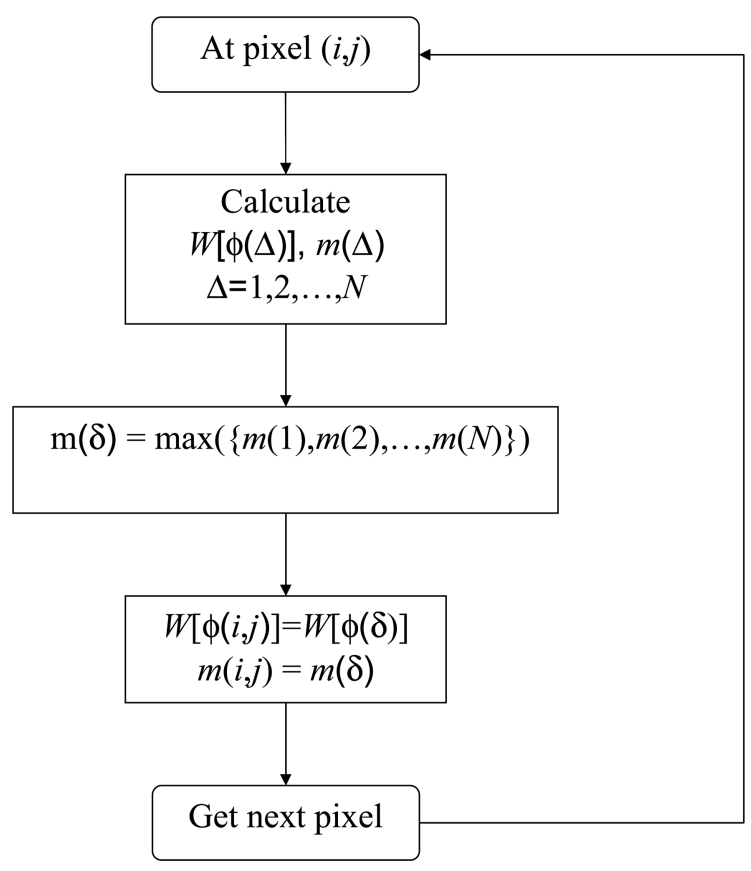

Fig. 2. Flow chart of the proposed algorithm. 
where $(x, y)$ range from 0 to 512 pixels, $r(x, y)$ is a Gaussian distributed noise and the phase is $\varphi(x)=\pi x^{2} / 1024$. Therefore the spatial frequency is $\omega=\pi x / 512$ and it changes linearly from 0 to $\pi \mathrm{rad} /$ pixel.

For each row, we have demodulated this signal using the adaptive asynchronous algorithm, with $\Delta=$ 6 pixels as the maximum decimating factor. We have also demodulated the signal using the standard fivestep asynchronous algorithm. For each point, we have calculated the response function using Eq. (3) and, afterward, we have averaged the modulation by columns. The results are shown in Fig. 3. The modulation responses appear noisy because we have computed them directly from the noisy data, not using the algebraic response expression given by Eq. (8). As expected, we can see that the adaptive algorithm presents a higher response for a wider range of frequencies than the standard one, so the phase recovery error will be lower. To show this, Fig. 4 depicts the phase recovery error for the adaptive and standard algorithms. For each row, we have computed the error between the ground truth and the obtained phase; afterward, we have averaged by columns. As can be seen in Fig. 4, globally the phase recovery error is lower for the adaptive algorithm, especially at the low and high frequencies. However, as is shown in Fig. 3 , the standard algorithm performs better than the adaptive one at the points where the decimation factor changes. This is due to the fact that, at these points, in the presence of noise the incorrect decimation factor can be selected due to the modulation fluctuation.

We have also compared our algorithm with the windowed Fourier ridge (WFR) technique using the MATLAB code provided in [8]. WFR is also an adaptive technique because it processes the fringe pattern locally in the frequency domain [8]. Compared with the

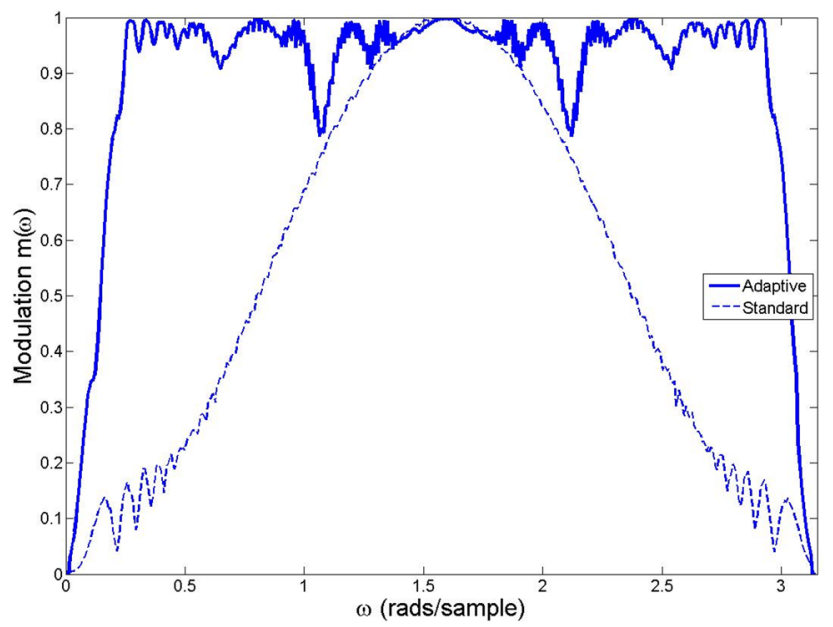

Fig. 3. (Color online) Response function for the adaptive five-step asynchronous algorithm measured from the noisy fringe pattern described in the text compared to that of the standard five-step asynchronous algorithm. The adaptive algorithm presents higher response for the low and high frequencies, which will lead to a more reliable phase demodulation for these frequencies.

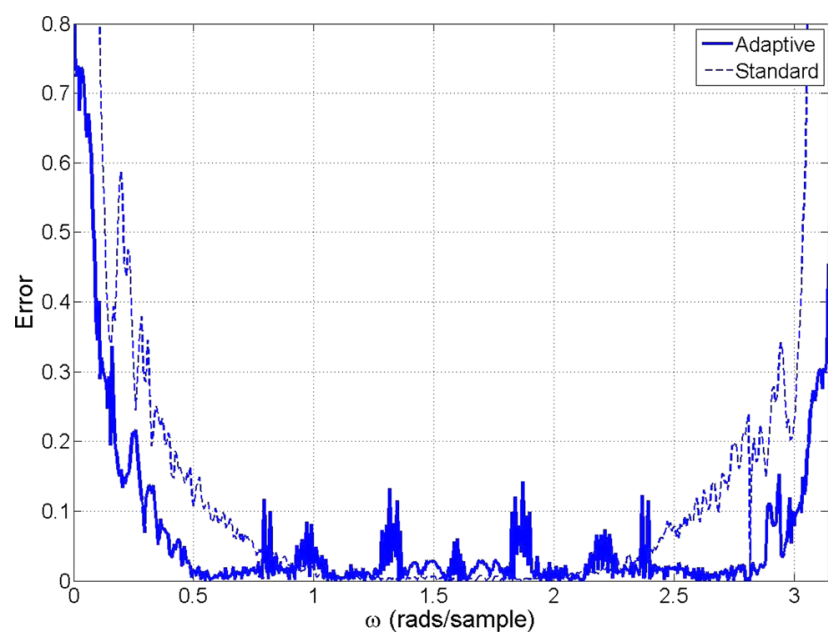

Fig. 4. (Color online) Comparison of the phase recovery error for the adaptive five-step asynchronous algorithm and the standard five-step asynchronous algorithm. As it could be expected from the response function of Fig. 3, the standard five-step algorithm behaves worse than the adaptive algorithm for low and high frequencies.

adaptive algorithm presented, the WFR technique has some disadvantages: First, for processing fringe patterns without a carrier, it is necessary to remove the background [8]. Second, WFR requires a priori estimation of the window size and range of frequencies; therefore, it is not very well suited for automatic processing. On the contrary, our technique only depends on the maximum decimation factor which is fixed by the desired modulation response. Finally, WFR is considerably slower than our algorithm. We have calculated the processing time needed to demodulate a $128 \times 128$ fringe pattern with the standard five-step algorithm, our adaptive technique, and the WFR. Using a computer with a $1.86 \mathrm{GHz}$ Core Duo processor and 2 gigabytes of RAM, we have obtained processing times of $0.03 \mathrm{~s}$ for the standard algorithm, $0.3 \mathrm{~s}$ for the adaptive algorithm, and $393 \mathrm{~s}$ for the WFR.

We have also tested our algorithm with real fringe patterns. In Fig. $\underline{5}$, we show a shadow-moiré fringe

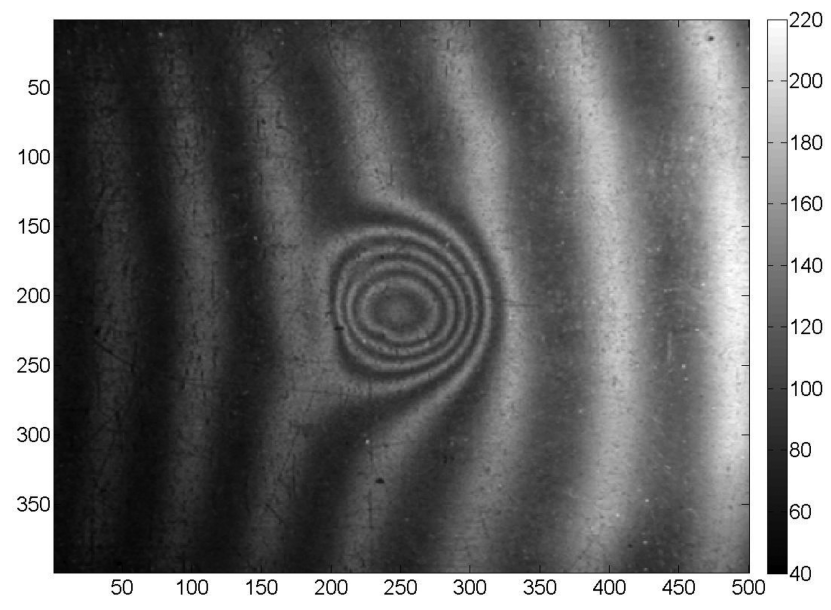

Fig. 5. Shadow-moiré fringe pattern of a smooth surface with a central indentation. 
pattern with nonuniform background and noise. We have demodulated this fringe pattern with our algorithm [Fig. 6(a)] and with the standard five-step asynchronous algorithm [Fig. 6(b)]. The selected fringe pattern presents a wide frequency content ranging from 7 fringes/field to 50 fringes/field in the central region. As can be seen in Fig. 6(a), the adaptive algorithm has correctly demodulated both the highand low-frequency portions of the fringe pattern, while the five-step asynchronous algorithm has failed to demodulate properly the low-frequency regions of the fringe pattern. The next step is to get the unwrapped phase. At this point, we must take into account that the asynchronous five-step algorithm when applied to closed fringe patterns presents a sign discontinuity for those points of the fringe pattern where the horizontal component of the spatial frequency is close to zero [see Figs. 6(a)-6(c)]. There are two solutions to this drawback: (1) we can change the experiment to introduce a carrier term on the fringe pattern or (2) we can correct the sign of the wrapped phase using this equation:

$$
\overline{W[\varphi]}=\operatorname{sign}[\cos (\theta)] W[\varphi],
$$

with $W[\varphi]$ as the wrapped phase resulting from the asynchronous algorithm, $W[\varphi]$ as the corrected

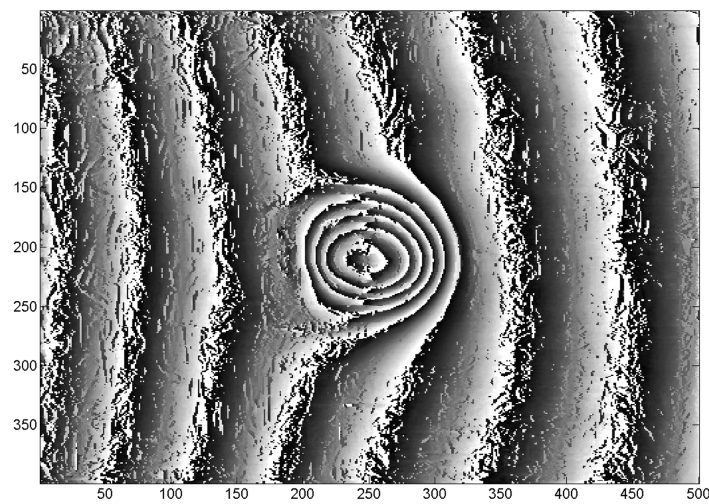

(a)

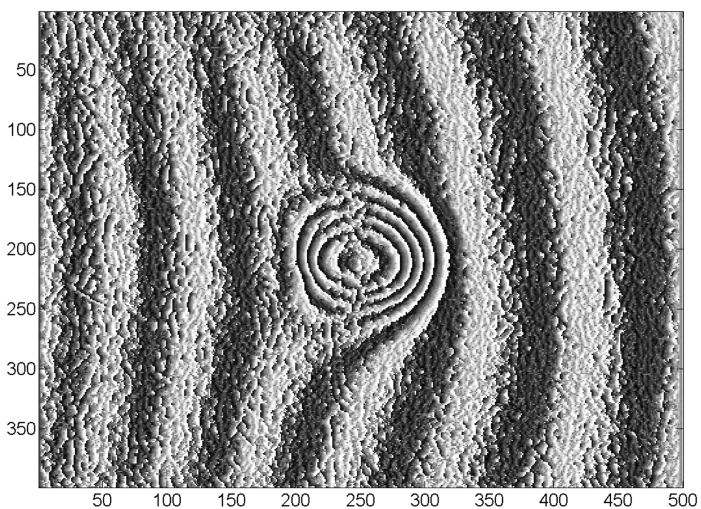

(b)

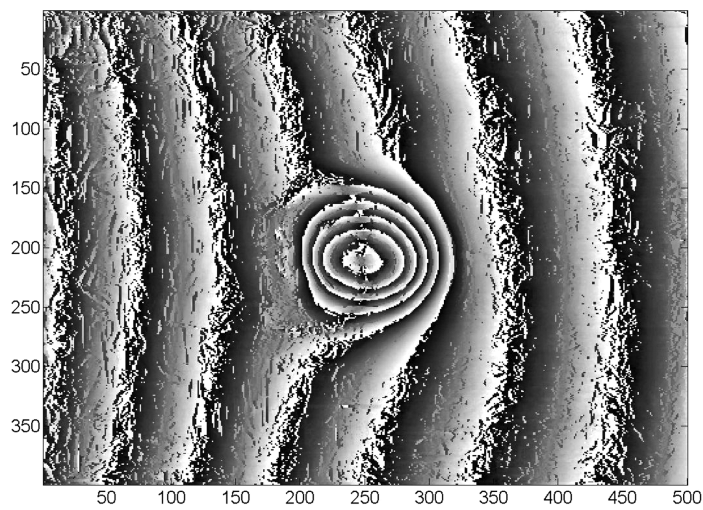

(c)

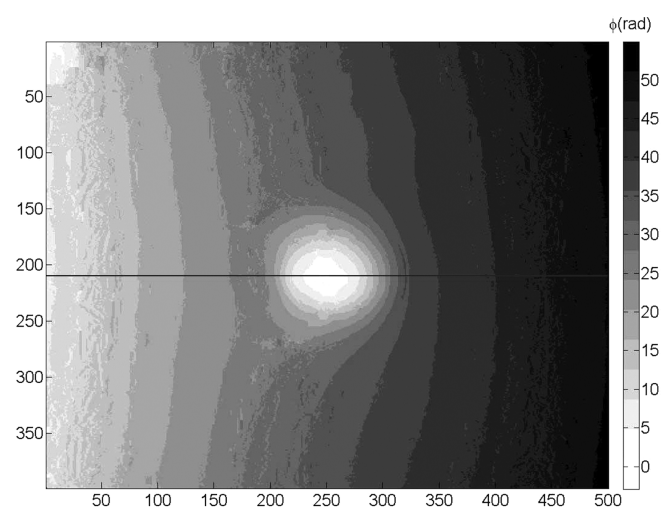

(d)

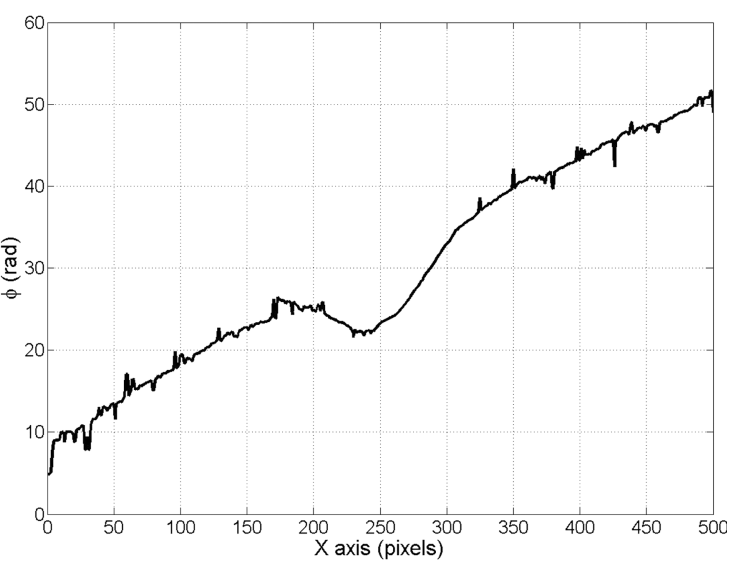

(e)

Fig. 6. (a) Wrapped phase of the fringe pattern of Fig. 5 recovered with the proposed method, (b) wrapped phase recovered with the standard five-step algorithm, (c) wrapped phase corrected through the fringe orientation, (d) unwrapped phase of the fringe pattern of Fig. $\underline{5}$, and (e) horizontal profile of the unwrapped phase along the 210th row of Fig. $\underline{6(d)}$. 
wrapped phase, and $\theta$ the direction of the fringes that has been calculated using the algorithm described in [11]. The corrected phase has been depicted in Fig. 6 $(\overline{\mathrm{d}})$, while the unwrapped phase has been depicted in Fig. 6(e). As can be seen in those figures, the sign discontinuity has been effectively removed.

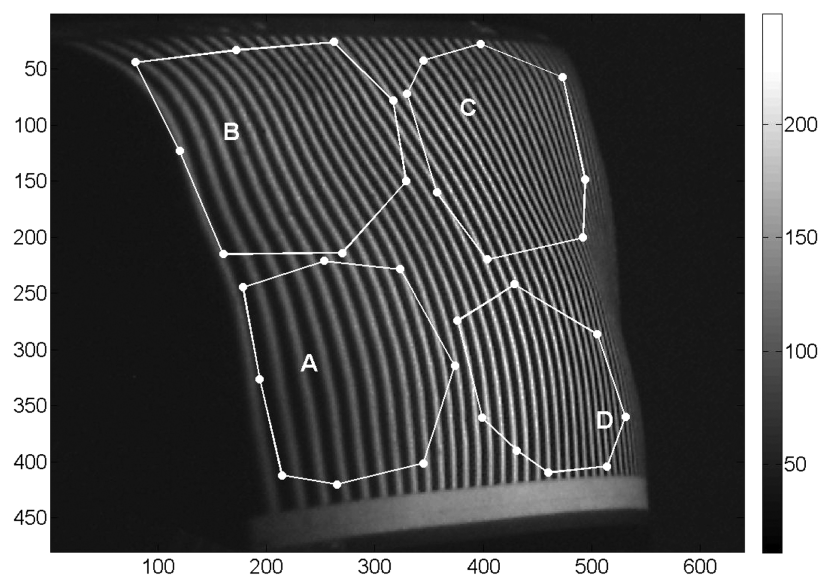

(a)

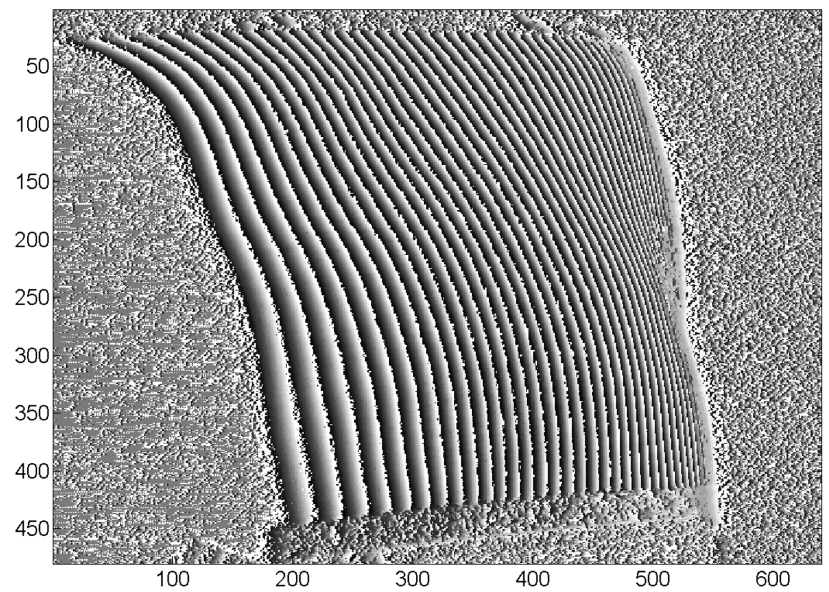

(b)

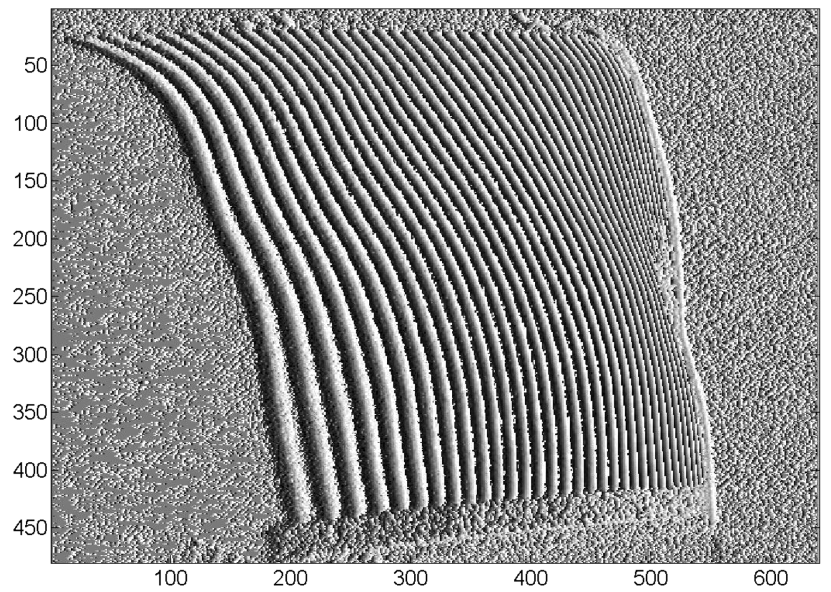

(c)

Fig. 7. (a) Fringe pattern projected over a human back, (b) wrapped phase recovered with the adaptive algorithm, and (c) wrapped phase recovered with the standard five-step algorithm.
Table 1. Standard Deviation of the Fit Error Obtained When Adjusting the Unwrapped Phase to a 3rd Degree Polynomial for the Adaptive and Standard Algorithms

\begin{tabular}{lccccc}
\hline & & Region A & Region B & Region C & Region D \\
\hline Adaptive & $\sigma_{\varphi}(\mathrm{rad})$ & 0.58 & 1.24 & 1.44 & 0.62 \\
Standard & $\sigma_{\varphi}(\mathrm{rad})$ & 2.20 & 1.86 & 1.93 & 1.35 \\
\hline
\end{tabular}

In Fig. 7 we present another example of the application of our algorithm. In this case, we have projected a sinusoidal fringe pattern over a human back [Fig. 7(a)]. The resulting fringe pattern presents a wide frequency variation across the field. We have demodulated this fringe pattern with our adaptive algorithm with a maximum decimation factor of $\Delta=6$ [Fig. 7(b)] and with the standard five-step algorithm [Fig. $7(\mathrm{c})]$. As can be seen in these figures, the adaptive algorithm has demodulated the phase correctly, independently of the spatial frequency, while the standard algorithm has not been able to deal properly with the low frequency regions of the fringe pattern.

To estimate the algorithm errors, we have unwrapped the phase and, afterward, we have selected four regions over the unwrapped phase map [labeled from A to D in Fig. 7(a)]. For each region, we have fitted the unwrapped phase to a 3rd degree polynomial and then we have computed the fit error as the difference between the unwrapped phase and the polynomial estimation. In Table 1 , we give the values of the standard deviation of the fit errors for the four regions represented in Fig. 7(a). In Fig. 8, we show the fit error histogram corresponding to region $\mathrm{A}$ of the phase map. As shown in this figure, the histogram corresponding to the adaptive algorithm is narrower than the one corresponding to the standard algorithm. Therefore, the fit error is lower for the adaptive algorithm. The same behavior is observed in the other regions of the phase map. Table 1 shows the calculated standard deviation of the fit error between the unwrapped phase and the approximating polynomial for each region depicted in Fig. 7(a).

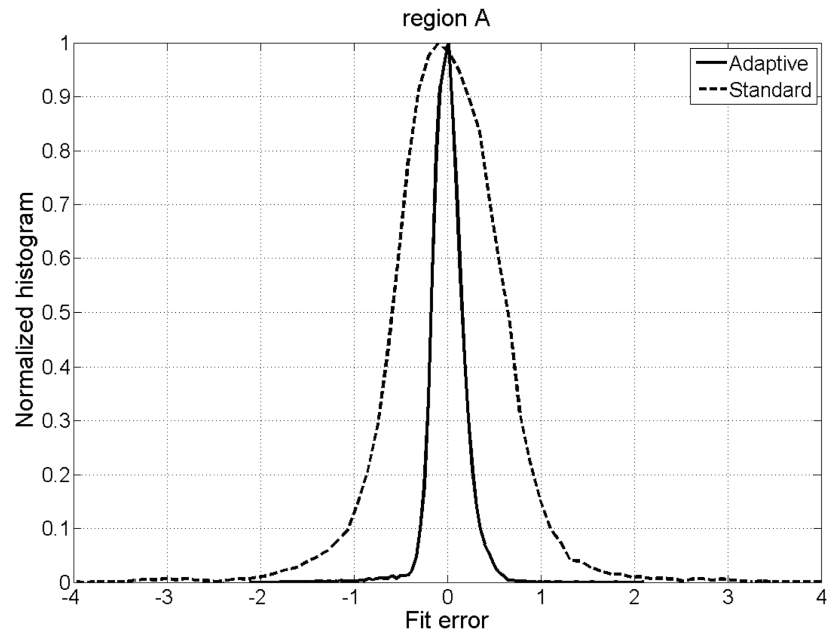

Fig. 8. Histogram of the fit error corresponding to the region labeled A in Fig. 7(a). 
Finally, we show a photoelastic fringe pattern in Fig. 9(a) with a wide frequency variation. This fringe pattern has been demodulated using the adaptive algorithm with $\Delta=6$ as the maximum decimation factor. In Fig. 9(b) we have represented the distribution of the values of the decimation factor $\Delta$ over the

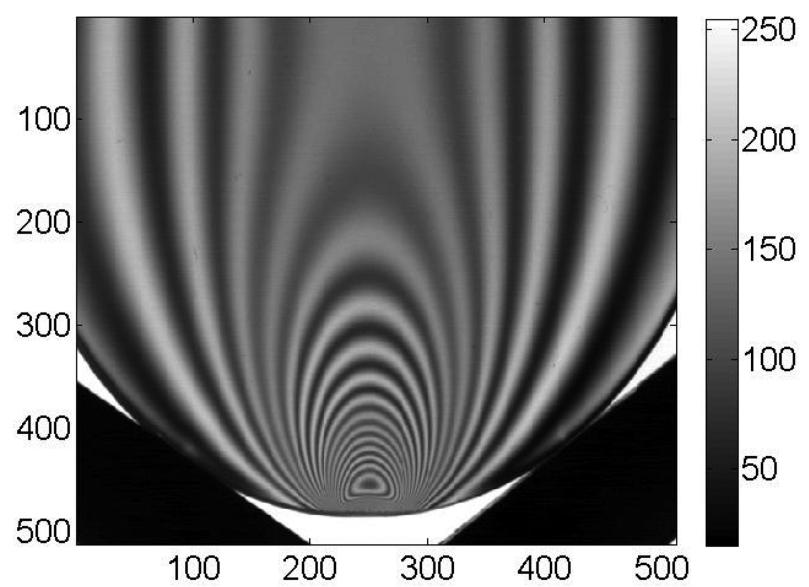

(a)

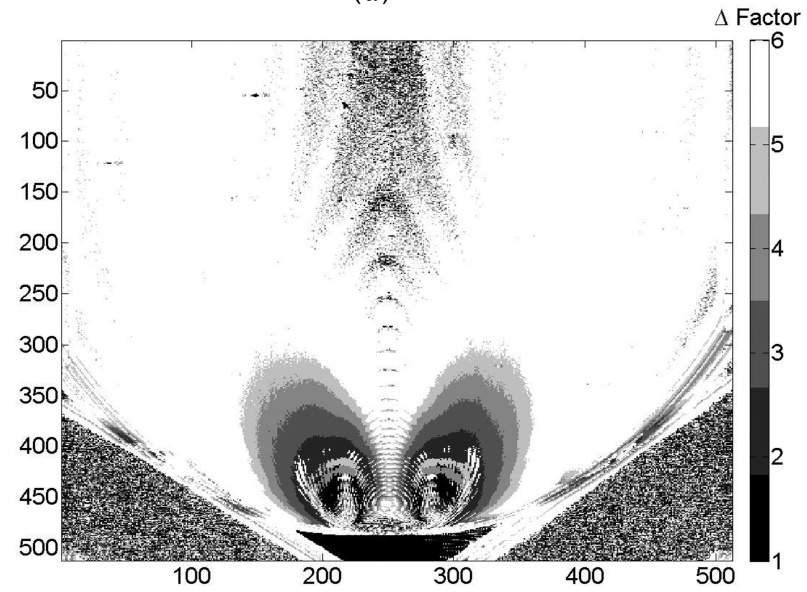

(b)

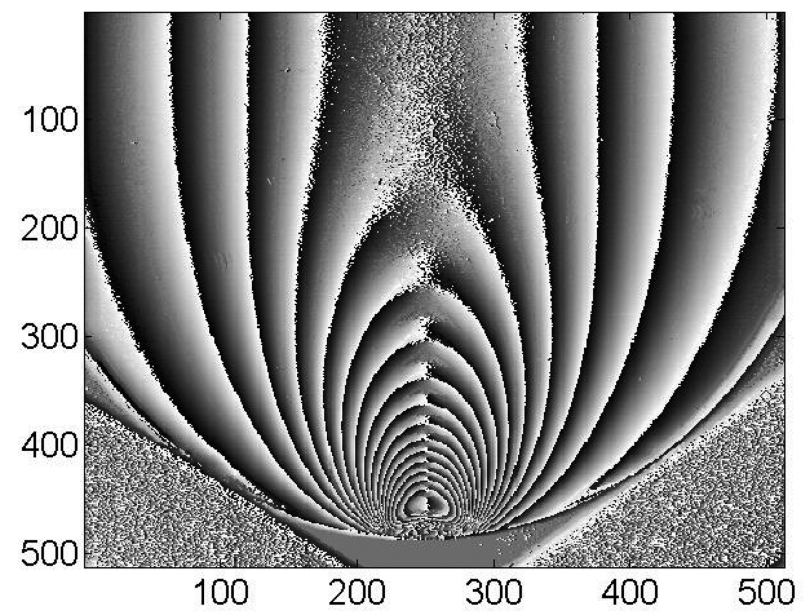

(c) fringe pattern. As expected from the frequency modulation of the adaptive algorithm (see Fig. 3), the decimation factor presents high values (around $\Delta=6$ ) when the local frequency is low, while it becomes lower when the local frequency is high. The resulting wrapped phase distribution is shown in Fig. $\underline{9(\mathrm{c})}$,

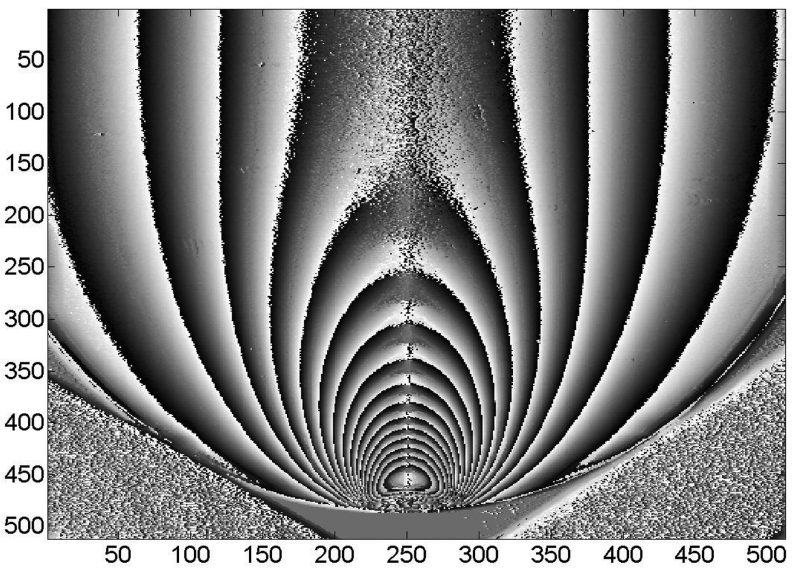

(d)

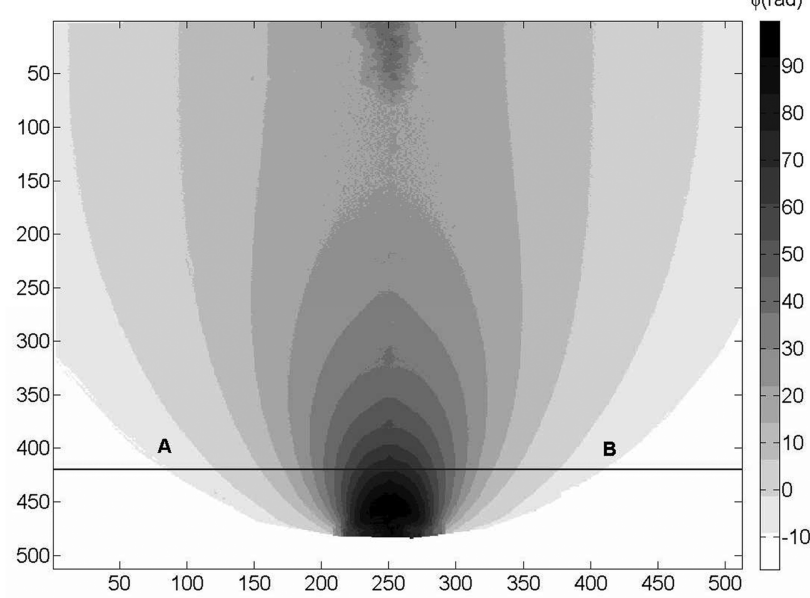

(e)

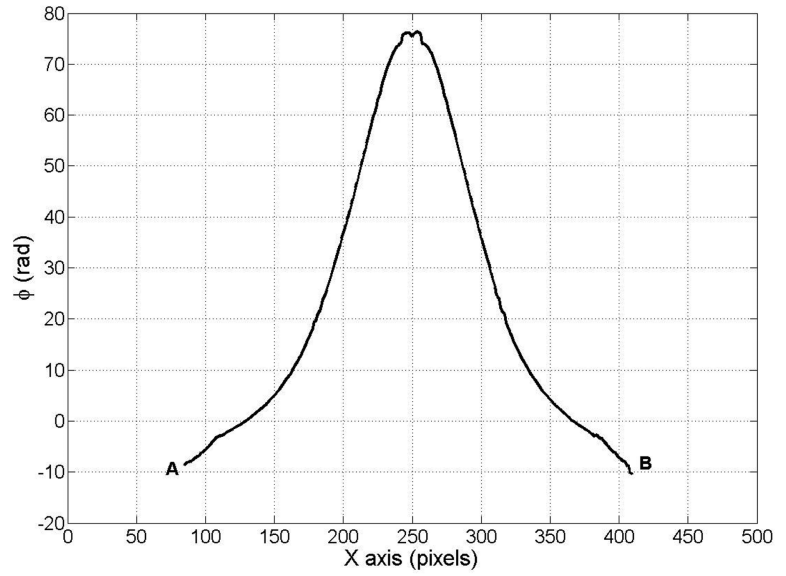

(f)

Fig. 9. (a) Experimental fringe pattern obtained in a photoelastic experiment, (b) distribution of the decimation factor over the fringe pattern, (c) wrapped phase recovered with the adaptive algorithm, (d) corrected wrapped phase, (e) unwrapped phase, and (f) horizontal profile of the continuous phase along points A and B of 9(e). 
the corrected wrapped phase is depicted in Fig. 9(d), and, finally, the unwrapped phase is shown in Fig. 9(e), where we can see that, despite the high frequency variation, the algorithm has been able to demodulate the phase properly even near the central vertical line where the horizontal spatial frequency is close to zero (see horizontal profile depicted in Fig. 9(e)]. We must point out, also, that the algorithm has been able to demodulate properly the phase, although there are fluctuations on the fringe modulation, which can be observed in Fig. 9(a). This means that our algorithm can deal with fringe patterns with modulation and background fluctuation whenever the spatial period of these variations is higher than the maximum size of the spatial domain where the algorithm works (in our case, a line of $2 \Delta+1$ pixel length); otherwise, we should carry a normalization of the fringe pattern before applying our algorithm.

\section{Conclusions}

We have presented a new technique for fringe pattern demodulation based on a standard five-step asynchronous phase sampling method, although it could be easily adapted to other standard asynchronous algorithms, such as Carré's. The proposed technique is based on a local decimation of the fringe pattern with an adaptive decimation factor selected in order to get the maximum response locally. Because of its adaptive nature, the proposed technique presents better frequency response than the standard algorithm, especially for the low and high spatial frequency regions. Compared with other adaptive techniques, such as the WFR, it is faster and more automatic because it does not require any adjustments of parameters, such as window size, range of frequencies, and so on. We have demonstrated the reliability of the proposed technique by demodulating both synthetic and experimental fringe patterns.
We acknowledge the financial support of this work by the Ministerio de Educación y Ciencia, project DPI2005-03891.

\section{References}

1. B. V. Dorrío and J. L. Fernández, "Phase-evaluation methods in whole-field optical measurement techniques," Meas. Sci. Technol. 10, R33-R55 (1999).

2. D. Malacara, M. Servín, and Z. Malacara, Interferogram Analysis for Optical Testing, 2nd ed. (CRC Press, 2005).

3. T. Kreiss, Holographic Interferometry (Akademie Verlag, 1996).

4. K. G. Larkin, "Efficient nonlinear algorithm for envelope detection in white light interferometry," J. Opt. Soc. Am. A 13, 832-843 (1996).

5. J. A. Gómez-Pedrero, J. A. Quiroga, and M. Servín, "Asynchronous phase demodulation algorithm for temporal evaluation of fringe patterns with spatial carrier," J. Mod. Opt. 51, 97109 (2004)

6. P. Carré, "Installation et utilisation du comparateur photoelectrique et interferentiel du Buerau International des Poids et Mesures," Metrologia 2, 13-23 (1966).

7. D. Crespo, J. A. Quiroga, and J. A. Gómez-Pedrero, "Design of asynchronous phase detection algorithms optimized for wide frequency response," Appl. Opt. 45, 4037-4045 (2006).

8. Q. Kemao Quian, "Windowed Fourier transform for fringe pattern analysis," Appl. Opt. 43, 2695-2702 (2004).

9. E. Berger, W. von der Linden, V. Dose, M. W. Ruprecht, and A. W. Koch "Approach for the evaluation of speckle deformation measurements by application of the wavelet transformation," Appl. Opt. 36, 7455-7460 (1997).

10. J. Zhong and J. Weng, "Spatial carrier-fringe pattern analysis by means of wavelet transform: wavelet transform profilometry," Appl. Opt. 43, 4993-4998 (2004).

11. J. Villa, I. De la Rosa, G. Miramontes, and J. A. Quiroga, "Phase recovery from a single fringe pattern using an orientational vector-field-regularized estimator," J. Opt. Soc. Am. A 22, 2766-2773 (2005). 